\title{
Fast Multi-area Approach for Distribution System State Estimation
}

\author{
C. Muscas, P. A. Pegoraro, S. Sulis \\ Department of Electrical and Electronic Engineering \\ University of Cagliari \\ Cagliari, Italy \\ (carlo, paolo.pegoraro, sara.sulis)@diee.unica.it
}

\author{
M. Pau, F. Ponci, A. Monti \\ Institute for Automation of Complex Power Systems \\ E.ON Energy Research Center, RWTH Aachen University \\ Aachen, Germany \\ (mpau, fponci, amonti)@eonerc.rwth-aachen.de
}

\begin{abstract}
This paper presents the application of a fast twostep multi-area approach for state estimation in wide-area distribution systems. In a previous paper [10], the authors have assessed the possibility to perform Distribution System State Estimation (DSSE) in a multi-area framework, designing the methodology according to the configuration of the measurement infrastructure. In this paper, constraints associated to the measurement placement are removed and the methodology proposed in [10] has been generalized to deal with every possible measurement system. A new second step procedure has been also designed to obtain a more computationally efficient refinement of the voltage profile. The proposed multi-area method can be performed in a decentralized way and with parallel processing, relying on limited data communication. Test results, obtained on the 123-bus IEEE test network, are presented and discussed.
\end{abstract}

Keywords-Distribution system state estimation, wide-area monitoring systems, multi-area state estimation, multi-area architecture

\section{INTRODUCTION}

Distribution systems, even under the deep changes that will affect the future power networks, are going to maintain their peculiarities with respect to transmission systems and, for this reason, their monitoring systems will present specific requirements and will require ad hoc Distribution System State Estimation (DSSE) techniques. In particular, it is unlikely that a complete monitoring of the network will be available and, thus, the number of measurement devices is expected to be very low. Besides, due to the introduction of new generation meter devices, such as phasor measurement units (PMUs) or smart meters (SMs), which are enabled with computation and communication features and are able to operate at faster reporting rate, the large size of distribution grids becomes a challenge for the design of fast and accurate DSSEs that rely on all the available information. Such information obviously includes the real-time measurements, but should also integrate all the available historical and forecast data, which can be continuously updated.

As aforementioned, it is unrealistic, from an economic point of view and for the feasibility of the measurement system maintenance, to expect that each node of the distribution network can be equipped with highly interconnected instrumentation. For this reason, it is essential that the DSSE is designed to estimate the state of the network, in terms of node voltages and branch currents, collecting measurement data from heterogeneous instruments with different accuracies and reporting rates. Thus, the DSSE has to deal with several measurement sources in a fast way, while keeping the estimation uncertainty below given limits, as required by the control center applications. In the literature, several DSSE techniques, mostly based on the weighted least squares (WLS) method, have been proposed [1]-[5]. Each method is designed to exploit specific characteristics of the distribution network or to include new measurement devices in a three-phase scenario (see, in particular, for PMUs [5]).

In a Smart Grid context, the topology of the distribution networks is changing from radial to more meshed, with bidirectional power flows due to distributed generation (DG), which make more complex the network monitoring aimed at Distribution Management System (DMS) applications [6]. Traditional control systems and application scheduling will no longer be useful in such an increasingly dynamic scenario, where an efficient and reliable control of active networks will be needed. An accurate knowledge of the network state obtained at high reporting rates, is needed for the new management applications to work correctly and with the necessary responsiveness.

In this regard, a relevant problem is that distribution systems usually include a large number of nodes and this aspect is emphasized by the three-phase nature of possibly unbalanced distribution networks. The new monitoring system, with an increasing number of meter points and the aggregation of heterogeneous information, should be able, for instance, to coordinate in "real-time" the DG and the distributed energy resources (DERs) or to track promptly highly dynamic behaviors. The meter configuration, the communication network and the computation infrastructure serving the DSSE and the overall monitoring system should be thought to cope with large amount of data and strict scheduling requirements.

For this reason, different multi-area approaches have been recently presented, in order to share the burden and the tasks of DSSE. In [7], a multi-area state estimation (MASE) approach based on a differential evolution algorithm is proposed. The main drawback is that local estimators need to exchange data with the adjacent sub-areas at each iteration of the estimation 
algorithm, thus asking for high communication and complex coordination. In [8], a multi-area method based on local WLS estimators is used. A central coordinator merging the results of the local estimations evaluates the voltage profile of the whole network. In [9] a comparison of simple two-step multi-area methods for distribution systems is performed and a possible multi-area architecture with shared measurement devices and overlapping areas is presented. In [10] the same architecture is used for a second step that takes into account the correlation of first step local estimations.

The multi-area DSSE proposed in [10] offers the advantages of parallel distributed computation and low communication costs, while speeding up the estimation process. In this paper, the same idea as in [10] has been followed; however, the constraints on the measurement infrastructure used in [10] have been removed and the design of the multi-area algorithm has been generalized in order to deal with every possible measurement system. Moreover, a more computationally efficient second step procedure, based on a fast WLS involving only one voltage estimation at a single node in each sub-area, is proposed. The new method is simpler and well-suited when measurement points are available at the overlapped nodes, at the border between different sub-areas. The following sections describe the considered multi-area architecture and the new proposed second step. Then, tests performed on the 123-bus IEEE distribution network, considering different measurement scenarios, are presented and discussed.

\section{MULTI-AREA DISTRIBUTION SYSTEMS STATE ESTIMATION}

Multi-Area State Estimation (MASE) methods are an important research topic in power systems since several decades. In transmission systems the major aims can be either to reduce the computing time and to exploit the characteristics of control centers distributed over the grid, or to integrate the estimation results of grids belonging to different Transmission System Operators (TSO) in order to enable Wide Area Monitoring Protection and Control (WAMPAC) [11]. In distribution systems, the major issue calling for scalable state estimation approaches is the large size of these networks. The main goal is thus to decompose the state estimation problem in order to lighten and to distribute the computational burden. An additional motivation is also given by the presence of grids operated at different voltage levels. In this case, a multi-area approach can allow separating the estimation for networks having different technical features.

Several MASE techniques have been proposed in literature, mainly devoted to transmission systems [12]-[15]. The various approaches can be distinguished according to different criteria, like the methodology of subdivision into areas, the type of estimator, the coordination scheme adopted in the multi-area harmonization, etc. Each approach can provide different advantages, for example on the accuracy of the estimation results, on the computational efficiency or as for the communication requirements. Despite the large number of proposals available for MASE in power systems, an efficient design of a MASE technique tailored to distribution systems is still a pending issue. It is worth noting that, in general, the methodologies available for the transmission systems cannot be directly applied at the distribution level because of the specific features of the distribution grids. Moreover, several aspects prevent an easy design of the multi-area approaches for distribution systems. The most important issue is the limited number of measurement devices available on the field. The lack of real-time measurements affects, first of all, the criteria for the partition of the whole grid in multiple sub-areas. In fact, a minimum set of measurements is required in each sub-area to guarantee the observability and to make the MASE process feasible. The scarcity of real-time measurements also implies, in general, the achievement of low accuracies through the estimation process. From this standpoint, the big challenge is to suitably distribute the state estimation problem among several sub-areas, while avoiding at the same time a detrimental degradation of the accuracy performance. Finally, the low number of measurement devices also influences the achievable robustness of the multi-area technique. In this case, it is clear that solutions aimed at obtaining minimum robustness requirements, for example against bad data measurements or communication failures, can restrict the set of viable multi-area configurations or schemes.

In general, the design of the multi-area DSSE approach has to find a proper trade-off between the conflicting requirements of efficiency, low computational burden and minimum data exchange on one side, and high accuracy performance and robustness on the other side. The multi-area approach proposed here aims at achieving such a compromise by means of a two step algorithm relying upon an architecture that can be implemented both in a centralized and in a decentralized way. Next sections provide more detailed information about the conceived multi-area architecture and the design of the two steps of the MASE algorithm.

\section{Multi-AREA DSSE PROPOSAL}

\section{A. Adopted multi-area architecture}

In this paper, the problem of distribution system state estimation is tackled by means of a multi-area framework based on the following assumptions:

- sub-areas should possibly have a similar number of nodes, in order to minimize the overall execution time avoiding the presence of a sub-area acting as a bottleneck during the parallel estimation runs;

- each sub-area must have as many measurements as needed to achieve its internal observability, even considering the possible presence of pseudomeasurements of load consumption or generator production;

- $\quad$ an overlapping node is assumed to be shared among adjacent sub-areas; this allows easy conversion of the electrical quantities exchanged between sub-areas when they refer to different phase-angle references, as it usually happens when conventional measurements are considered; additional benefits can be also present when measurement points are placed at the overlapping node, as in [10];

- the computing architecture can be decentralized and the coordination scheme is at SE level; this means that each 
sub-area needs to exchange data only with the neighboring sub-areas and the communication is carried out at the end of the first step local estimation.

The estimation procedure is performed in two main steps, which correspond to a local estimation in each sub-area followed by a fast harmonization of the results exploiting the border information coming from the adjacent sub-areas. With respect to the approach already proposed in [10], all the constraints regarding the measurement infrastructure are here removed; the only assumption is that a sufficient number of measurements is available in order to guarantee the observability of each sub-area.

In the following, the two steps of the MASE procedure are described in detail, along with the assumptions. For the sake of clearness, the description focuses on the sub-area A of the unbalanced IEEE 123-bus test network, considering the partition reported in Fig. 1. Such network is also used for the tests in the Section IV.

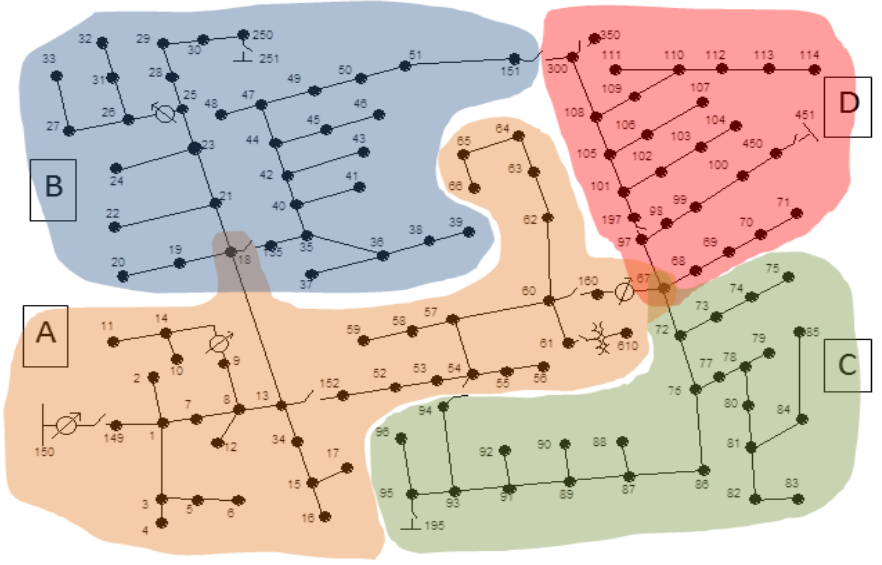

Fig. 1. IEEE 123-bus test network: organization in sub-areas.

\section{B. Local estimations}

The starting step of the multi-area approach is the parallel execution of a local state estimation in each one of the subareas that compose the DSSE architecture. The inputs, for each one of the local estimations, are the real-time, pseudo- and virtual measurements (e.g. zero injection measurements). If available, real-time measurements at the overlapped nodes are also included in the set of input measurements. As shown in [10], these can include the voltage measurement and an equivalent power injection measurement at the overlapped node (the latter is calculated by summing up the power measurements at the branches converging to the overlapped node that do not belong to the considered sub-area).

The SE algorithm used to perform the local estimations is the three-phase version of the WLS Branch-Current Distribution System State Estimator (BC-DSSE) presented in [5]. This allows for an easy integration of any kind of measurement and for high execution speed, without any loss of accuracy [16]. According to [5], the state vector $\mathbf{x}$ includes the bus voltage of a reference node as well as the branch currents in rectangular coordinates. Such a choice of the state vector allows, beyond the direct estimation of the state variables, also the computation of the whole voltage profile of the sub-area (through the calculation of the voltage drops associated to the estimated currents, starting from the estimated reference bus voltage). It is worth noting that the WLS algorithm also allows calculating the covariance matrix associated to the estimated states: this can be obtained through the inversion of the socalled Gain matrix used during the estimation process. Starting from such covariance matrix, the uncertainty associated to the different node voltages can be also found applying the uncertainty propagation law.

As a result, the output of each sub-area at the end of the first step is the set of branch currents and node voltages, plus the covariance matrices of the estimated states and of the node voltages.

\section{Estimation harmonization}

The second step is performed in each subarea by collecting the necessary data coming from the first step estimations of the adjacent sub-areas, and using them to refine the estimation results through a second WLS algorithm. Two different WLS procedures are considered in this paper for the second step. The first one, as described in [10], is based on a second state estimation on the entire sub-area that integrates the previous estimated states with the border information provided by the adjacent sub-areas. The second WLS method here proposed, instead, aims at achieving better computational performance and only focuses on the refinement of the voltage profile. It is based on a mini-WLS that includes the estimated reference node voltage of the considered sub-area and its estimates obtained from the voltage estimates of neighboring zones. More in detail, both methods can be described through the sequence of the following passages:

- collection of the necessary data from the adjacent subareas;

- creation of the set of input measurements for the second step WLS algorithm;

- creation of the covariance matrix for the overall set of input measurements;

- execution of the second step WLS estimation.

Since the first WLS procedure has been already presented in [10], in the following the focus will be mainly on the description of the new WLS algorithm. When necessary, reference is made to the first solution mainly to highlight the benefits brought by the new method from a computational point of view.

In the new algorithm, the data needed from each adjacent sub-area are limited to the voltage estimation at the overlapped node and its variance. Thus, a very low communication cost is required by the harmonization process. Referring to Fig. 1 and considering for example sub-area $\mathrm{A}$, if a measurement system composed of traditional devices (i.e. without synchrophasors measured by PMUs) is assumed, the data needed by the second step algorithm are the three-phase voltage magnitudes $\mathbf{V}_{18 \mathrm{~B}}, \mathbf{V}_{67 \mathrm{C}}$ and $\mathbf{V}_{67 \mathrm{D}}$ on nodes 18 and 67 (provided by subareas $\mathrm{B}, \mathrm{C}$ and $\mathrm{D}$, respectively) and their corresponding uncertainties. Note that the approach proposed in [10] also 
requires an equivalent power injection at the overlapped node, together with its uncertainty.

To obtain the set of input measurements in the new method, differently from [10], the estimations obtained from the neighbouring sub-areas have to be post-processed. In particular, the voltages at the overlapped node are propagated to the reference bus of the considered sub-area, in order to get a set of input measurements all referring to the same bus. Considering the example in Fig.1, if the reference bus of subarea $\mathrm{A}$ is node 1 , the set of input measurements used in the second step would be: $V_{1 A}, V_{1 B}, V_{1 C}$ and $V_{1 D}$, where $V_{1 A}$, is the voltage at node 1 estimated in the sub-area $A$, while $\mathbf{V}_{1 \mathrm{~B}}, \mathbf{V}_{1 \mathrm{C}}$ and $\mathbf{V}_{1 \mathrm{D}}$ are the three-phase voltage magnitudes derived from the voltages $\mathbf{V}_{18 \mathrm{~B}}, \mathbf{V}_{67 \mathrm{C}}$, and $\mathbf{V}_{67 \mathrm{D}}$, i.e. from the voltages estimated in subareas $\mathrm{B}, \mathrm{C}$ and $\mathrm{D}$, respectively, in the nodes shared with the sub-area A. This calculation can be made by considering the voltage drops given by the branch currents estimated during the first step. As an example, the complex voltage at phase $p$ in node 1 due to the sub-area B voltage estimate is:

$$
v_{1 \mathrm{~B}}^{p}=v_{18 \mathrm{~B}}^{p}+\sum_{\mathrm{j} \in \Gamma_{1,18}} \mathbf{z}_{j}^{p} \mathbf{i}_{j}
$$

where $v_{1 \mathrm{~B}}^{p}$ indicates the voltage phasor, while $\mathbf{z}_{j}^{p}$ and $\mathbf{i}_{j}$ represent the $p$-th row of the complex impedance matrix and the current phasors vector on the $j$-th branch in the path between node 1 and node 18 (the set $\Gamma_{1,18}$ ). It is worth noting that in case of measurement system with traditional devices, since the phase angle references of sub-area A and B are different, the voltage phasor $v_{18 \mathrm{~B}}^{p}$ to be considered is:

$$
v_{18 \mathrm{~B}}^{p}=V_{18 \mathrm{~B}}^{p} \cdot e^{j \theta_{18 \mathrm{~A}}^{p}}
$$

which means that the considered voltage phase-angle in node 18 is the one calculated through the local state estimation of sub-area $\mathrm{A}$ (the estimated phase-angle is $\left.\theta_{18 \mathrm{~A}}^{p}\right)$. This is equivalent to make a shift in the phase-angle reference of subarea $\mathrm{B}$, in order to assume the same phase-angle reference used in the considered sub-area A. Equation (1) can be rewritten then for the voltage magnitude as:

$$
\begin{aligned}
V_{1 \mathrm{~B}}^{p}= & V_{18 \mathrm{~B}}^{p} \cdot \cos \left(\theta_{18 \mathrm{~A}}^{p}-\theta_{1 \mathrm{~A}}^{p}\right) \\
& +\sum_{\mathrm{j} \in \Gamma_{1,18}} \sum_{k=1}^{3} a_{j}^{p k}\left(i_{j}^{r}\right)_{k}+b_{j}^{p k}\left(i_{j}^{x}\right)_{k}
\end{aligned}
$$

where the subscript $k$ scans the phase indices and, given the generic impedance element $z_{j}^{p k}=\left(R_{j}^{p k}+j X_{j}^{p k}\right)$ for branch $j$ :

$$
\begin{aligned}
& a_{j}^{p k}=R_{j}^{p k} \cdot \cos \left(\theta_{1 \mathrm{~A}}^{p}\right)+X_{j}^{p k} \cdot \sin \left(\theta_{1 \mathrm{~A}}^{p}\right) \\
& b_{j}^{p k}=R_{j}^{p k} \cdot \sin \left(\theta_{1 \mathrm{~A}}^{p}\right)-X_{j}^{p k} \cdot \cos \left(\theta_{1 \mathrm{~A}}^{p}\right)
\end{aligned}
$$

This type of equations are thus used to calculate the equivalent voltage magnitude estimations in the reference node of the considered sub-area, and such quantities are used as equivalent measurements for the WLS algorithm of the multi-area second step.
To apply a WLS to the set of obtained equivalent measurements, the last step is the computation of the input measurements covariance matrix. This matrix can be computed by means of the uncertainty propagation law, according to the following:

$$
\boldsymbol{\Sigma}^{(2)}=\left[\frac{\partial \mathbf{f}}{\partial \mathbf{x}^{\mathrm{T}}}\right]\left[\boldsymbol{\Sigma}^{(1)}\right]\left[\frac{\partial \mathbf{f}}{\partial \mathbf{x}^{\mathrm{T}}}\right]^{\mathrm{T}}
$$

where $\boldsymbol{\Sigma}^{(1)}$ is the covariance matrix of the first step estimates vector $\mathbf{u}=\left[\mathbf{V}_{1 \mathrm{~A}}^{\mathrm{T}}, \mathbf{V}_{18 \mathrm{~B}}^{\mathrm{T}}, \mathbf{V}_{67 \mathrm{C}}^{\mathrm{T}}, \mathbf{V}_{67 \mathrm{D}}^{\mathrm{T}}, \mathbf{i}_{\mathrm{A}}^{\mathrm{r}}{ }^{\mathrm{T}}, \mathbf{i}_{\mathrm{A}}^{\mathrm{x}^{\mathrm{T}}}\right]^{\mathrm{T}}$ used to calculate the derived measurements $\left(\mathbf{i}_{\mathrm{A}}^{\mathrm{r}}\right.$ and $\mathbf{i}_{\mathrm{A}}^{\mathrm{X}}$ are the vector including all the real and imaginary parts of the branch currents in area A) and $\frac{\partial \mathbf{f}}{\partial \mathbf{x}^{T}}$ is the Jacobian of the vector function $\mathbf{f}$, which includes the equations used to calculate each output measurement (eq. (3) in the example). In (6), it is possible to observe that the starting covariance matrix $\Sigma^{(1)}$ includes the covariance matrix of the local BC-DSSE output obtained after the first step and a covariance sub-matrix associated to the voltage magnitudes estimations in the overlapped nodes provided by the adjacent sub-areas. In [10], the importance of taking into account possible correlations arising among the voltage estimations coming from different sub-areas was proved. According to the mathematical analysis performed in [10], these correlations exist if shared voltage measurements are present at the overlapped node. In this case, the resulting covariance terms in $\boldsymbol{\Sigma}^{(1)}$ have to be duly considered and calculated through the relationships shown in [10].

Finally, the second step of the multi-area state estimation performs an additional WLS estimation of the reference bus voltage by using the equivalent measurements calculated by (3) and the inverse of the covariance matrix $\boldsymbol{\Sigma}^{(2)}$, obtained in (6), as weighting matrix. When considering traditional measurements, the aim of the second step estimation is only the refinement of the reference bus voltage magnitude. As a consequence, the WLS estimates only three state variables, one for each phase of the system. All the equivalent measurements provided as input are voltage magnitudes, and thus the Jacobian of the measurement functions is a column vector of ones. After the WLS, the final value of the reference bus (for example, node 1 for area A) voltage magnitude is obtained. Then, the voltage profile of the whole sub-area can be refined by computing the voltage drops given by the branch current estimations of the first step local BC-DSSE.

As it can be observed, with respect to the methodology proposed in [10], this new method involves a more complex preparation of the set of input measurements and of the corresponding covariance matrix. Nevertheless, the following WLS algorithm is linear and only has to estimate one state variable per phase, differently from the WLS algorithm in [10] where the entire state estimation process has to repeated. The main drawback of this new approach is that it does not allow the refinement of the current estimations. However, as it will be shown in the following tests, there can be particular scenarios where an additional current estimation is not necessary and therefore the use of this methodology can result convenient. 


\section{TESTS AND RESUlTS}

All the tests presented in this section have been performed using the three phase unbalanced IEEE 123-bus test network (Fig. 1). Data about the network are available in [17]. The results obtained with the following three measurement configurations will be discussed:

1. Case 1: measurement points only on non-overlapped nodes, and, in particular, on nodes $1,25,152,76,101$.

2. Case 2: measurement points only on the overlapped nodes $1,18,67$.

3. Case 3: measurement points on both overlapped and nonoverlapped nodes $(1,18,25,152,67,76,101)$.

Each considered measurement point includes a voltage magnitude measurement at the node and power flow measurements at all the branches connected to the node. Pseudo-measurements of power injections are assumed to be known for all the load nodes. With the chosen assumptions, all the used measurement configurations guarantee full observability for each sub-area. Monte Carlo simulations (25000 trials for each test) have been used to evaluate the performance of the proposed multi-area state estimation methods. For each trial, the "reference" values of the electrical quantities in the network are obtained by means of a load flow calculation. Then, measurements are randomly extracted according to their probability density function. In particular, a Gaussian distribution has been used for both real-time measurements and pseudo-measurements. The standard deviations are assumed equal to one third of $50 \%$ of the nominal value for the pseudo-measurements and of $1 \%$ and $3 \%$ of the actual value for voltage magnitude and power flow measurements, respectively.

In the following, the performance of the proposed approach for multi-area state estimation, in both the formulation discussed in [10] (indicated as MA-DSSE, in the following) and the new version here proposed (MA-DSSE new, in the following) is compared to the results of DSSE carried out on the whole network (Integrated State Estimation, ISE). Reported results only refer to one phase of the threephase system, but identical considerations apply to all the phases of the system. It is worth noting that the number of nodes in each phase is different from the total number of nodes, because of the presence of some branches that are not three-phase. The numbering of the nodes is increasing, following Fig. 1, but the node subsets corresponding to the four subareas are clearly indicated.

The first test has been performed to verify the goodness of the proposed multi-area architecture when considering a nonconstrained measurement configuration. Fig. 2 reports the results in terms of Root Mean Square Error (RMSE) for the voltage magnitude estimation of each node. It can be observed that, as also shown in [10], the MA-DSSE methods reach the same accuracy as ISE when the effect of all the measurement devices can be "included" in the second step (as it occurs in this case only for sub-area A). It is also interesting to note that, despite its increased efficiency, the MA-DSSE-new achieves the same accuracy as the MA-DSSE method. However, such advantages are obtained at the expense of the accuracy in the current estimations at some nodes near the subarea borders. This can be observed in Fig. 3, where the RMSE values for the current magnitude estimations are reported.

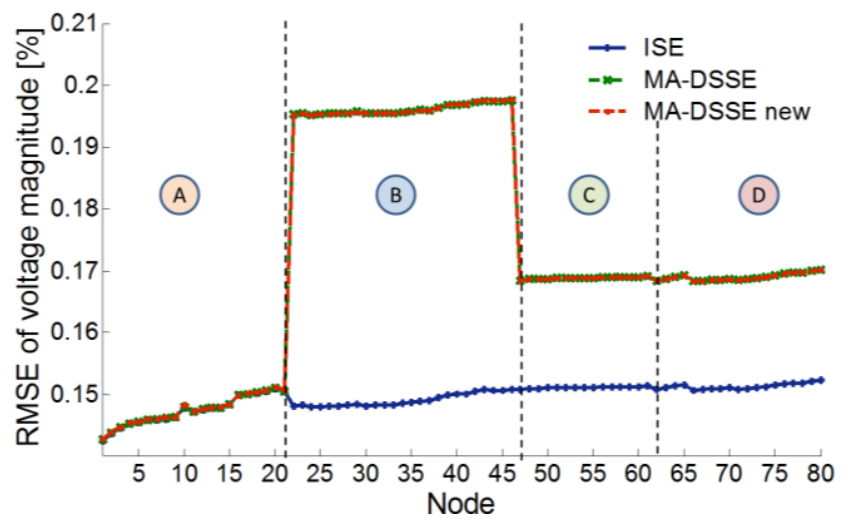

Fig. 2. RMSE of voltage magnitude estimations with Case 1 measurement system.

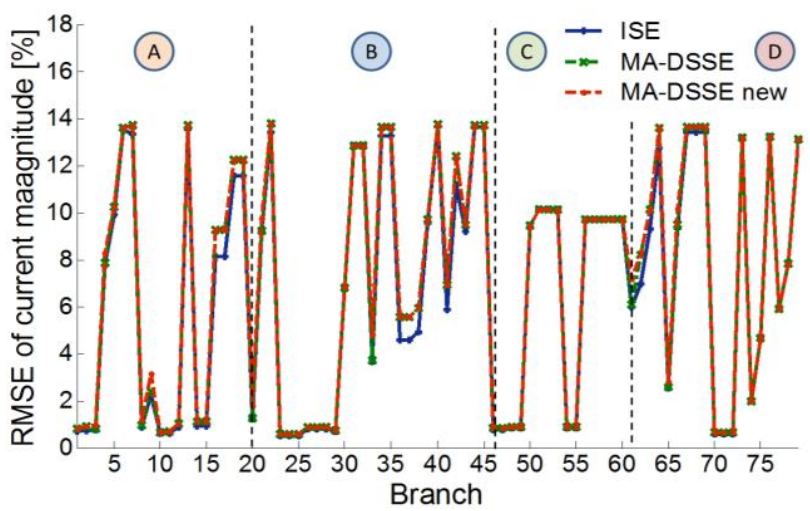

Fig. 3. RMSE of current magnitude estimations with Case 1 measurement system.

When considering Case 2 and Case 3 measurement systems, MA-DSSE new and MA-DSSE methods have still similar results for the voltage amplitude profile, as in the previous case (shown in Fig. 4 for Case 2). Besides, in these cases, also the RMSE results for current amplitude estimation are almost the same for MA-DSSE new and MA-DSSE (and also with respect to ISE, as a main difference with respect to the non-constrained Case 1), as shown in Fig. 5 for Case 3. This confirms the role of the shared devices in decoupling the subarea local estimations and thus supports the usefulness of MA-DSSE new both in voltage and current estimation when the characteristics of the measurement system are fit. 


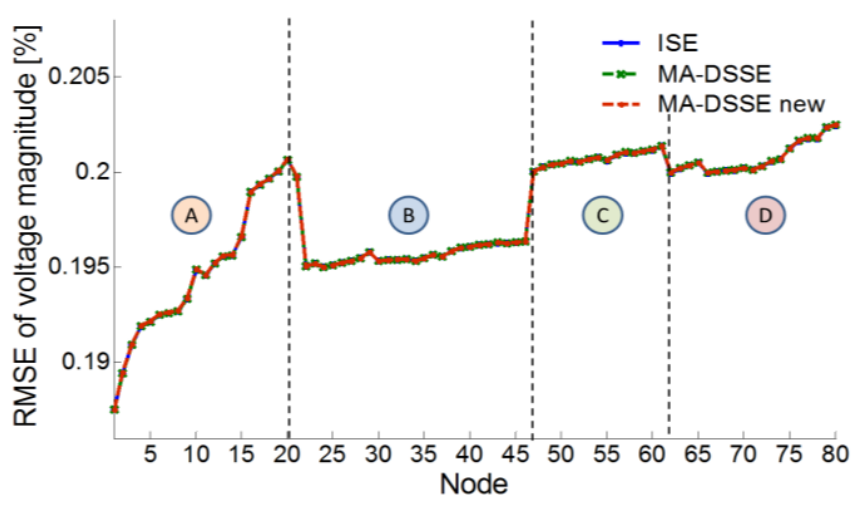

Fig. 4. RMSE of voltage magnitude estimations with Case 2 measurement system.

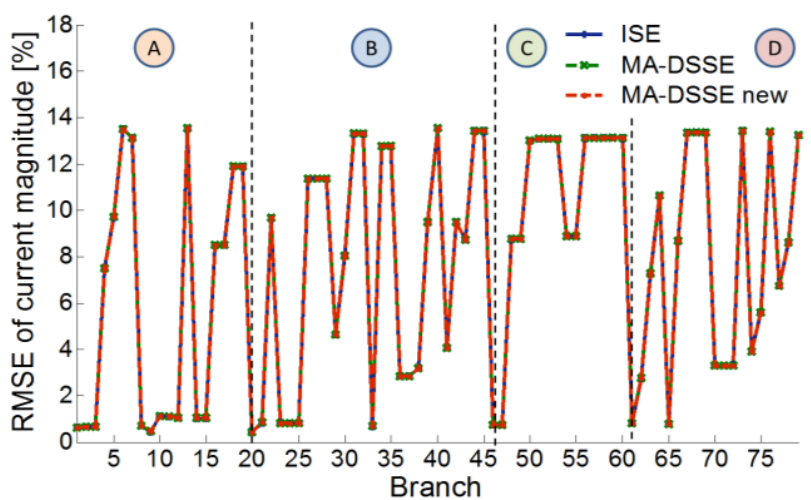

Fig. 5. RMSE of current magnitude estimations with Case 3 measurement system.

\section{CONCLUSION}

The paper proposes an architecture for multi-area distribution systems state estimation subject to no particular constraints in the measurement system configuration. A new simplified and faster approach to the second step of the estimation is also introduced. The test results show that such approach can be profitably applied to the voltage profile estimation, irrespective of the chosen measurement configurations, while it is particularly useful also for current estimations when the measurement system is designed with shared measurements at the overlapping nodes.

\section{ACKNOWLEDGMENT}

This work was supported by Regione Autonoma della Sardegna, L.R. 7/2007: "Promozione della ricerca scientifica e dell'innovazione tecnologica in Sardegna, annualità 2012”, CRP-60511.

\section{REFERENCES}

[1] M. E. Baran and A. W. Kelley, "State estimation for real-time monitoring of distribution systems," IEEE Trans. Power Syst., vol. 9, no. 3, pp. 1601-1609, Aug. 1994.

[2] W. M. Lin and J. H. Teng, "Distribution fast decoupled state estimation by measurement pairing," IEEE Proceedings - Generation, Transmission and Distribution, vol. 143, no. 1, pp. 43-48, Jan. 1996.
[3] M. E. Baran and A. W. Kelley, "A branch-current-based state estimation method for distribution systems," IEEE Trans. Power Syst., vol. 10, no. 1, pp. 483-491, Feb. 1995.

[4] H. Wang; N.N.Schulz, "A revised branch current-based distribution system state estimation algorithm and meter placement impact," IEEE Trans. Power Syst., vol.19, no.1, pp.207-213, Feb. 2004.

[5] M. Pau, P.A. Pegoraro and S. Sulis, "Efficient Branch-Current-Based Distribution System State Estimation Including Synchronized Measurements," IEEE Trans. Instrum. Meas., vol. 62, no. 9, pp. 24192429, Sep. 2013.

[6] Celli, G.; Pegoraro, P.A.; Pilo, F.; Pisano, G.; Sulis, S., "DMS CyberPhysical Simulation for Assessing the Impact of State Estimation and Communication Media in Smart Grid Operation," IEEE Trans. Power Syst., vol.29, no.5, pp.2436-2446, Sept. 2014.

[7] N. Nusrat, M. Irving and G. Taylor, "Development of distributed state estimation methods to enable smart distribution management systems," 2011 IEEE International Symposium on Industrial Electronics (ISIE), pp. 1691-1696, Jun. 2011.

[8] L. De Alvaro Garcia and S. Grenard, "Scalable distribution state estimation approach for Distribution Management Systems," $20112^{\text {nd }}$ IEEE PES International Conference and Exhibition on Innovative Smart Grid Technologies (ISGT), pp. 1-6, Dec. 2011.

[9] C. Muscas, M. Pau, P. A. Pegoraro, S. Sulis, F. Ponci, A. Monti, "Twostep procedures for wide-area Distribution System State Estimation," in Proceeding of IEEE International Instrumentation and Measurement Technology Conference (I2MTC), Montevideo, Uruguay, May 2014, pp.1517-1522.

[10] C. Muscas, M. Pau, P. A. Pegoraro, S. Sulis, F. Ponci, A. Monti, "Multiarea Distribution System State Estimation,"IEEE Trans. Instrum. Meas., vol.64, no.5, pp.1140-1148, May 2015.

[11] A. Gomez-Exposito, A. de la Villa Jaen, C. Gomez-Quiles, P. Rosseaux and T. Van Cutsem, "A Taxonomy of Multi-Area State Estimation Methods," Electric Power Systems Research, vol. 81, no. 4, pp. 1060 1069, 2011.

[12] T. Van Cutsem, J. L. Horward and M. Ribbens-Pavella, "A two level static state estimator for electric power systems," IEEE Transaction on Power Apparatus and Systems, vol. PAS-100, no. 8, pp. 3722-3732, Aug. 1981.

[13] D. M. Falcao, F. F. Wu and L. Murphy, "Parallel and distributed state estimation," IEEE Trans. Power Syst., vol. 10, no. 2, pp. 724-730, May 1995.

[14] L. Zhao and A. Abur, "Multiarea State Estimation Using Synchronized Phasor Measurements," IEEE Trans. Power Syst., vol. 20, no. 2, pp. 611-617, May 2005.

[15] A. J. Conejo, S. de la Torre and M. Canas, "An Optimization Approach to Multiarea State Estimation," IEEE Trans. Power Syst., vol. 22, no. 1, pp. 213-221, Feb. 2007.

[16] M. Pau, P.A. Pegoraro and S. Sulis, "Performance of Three-Phase WLS Distribution System State Estimation Approaches", IEEE Int. Workshop on Appl. Meas. for Power Systems (AMPS), Aachen, Germany, Sep. 2015 .

[17] (2012) IEEE test feeder specifications. [Online]. Available: http://ewh.ieee.org/soc/pes/dsacom/testfeeders/ 